\title{
Energetics and Electronic Structure of Armchair Nanotubes with Topological Line Defect
}

\author{
Susumu Okada ${ }^{1}, K_{y}$ oko Nakada ${ }^{2}$, Takazumi Kawai ${ }^{3}$ \\ ${ }^{1}$ Institute of Physics and Center for Computational Sciences, University of Tsukuba, \\ Tennodai, Tsukuba 305-8571, Japan \\ and CREST, Japan Science and Technology Agency, 4-1-8 Honcho, Kawaguchi, \\ Saitama 332-0012, Japan \\ ${ }^{2}$ Department of Chemistry and Biological Science, Aoyama Gakuin University, \\ Fuchinobe, Sagamihara 229-8558, Japan \\ ${ }^{3}$ Fundamental and Environmental Research Laboratories, NEC Corporation, 34 \\ Miyukigaoka, Tsukuba 305-8501, Japan \\ E-mail: sokada@comas.frsc.tsukuba.ac.jp
}

\begin{abstract}
We study electronic structure and energetics of carbon nanotubes with topological line defect consisting of fused pentagons and octagon rings by using the first-principles calculation in the density functional theory and tight-binding molecular dynamics simulations. The tubes with the topological line defects are found to exhibit magnetic ordering that polarized electron spins are localized around the topological defect and ferromagnetically aligned along the defect. Our analyses on the electronic energy band and spin density distribution reveal that this ferromagnetic spin ordering is associated with the edge states that is inherent in the graphite ribbon with zigzag edges. The tight-binding molecular dynamics simulations show that the nanotubes with the topological line defects are thermally stable up to temperature of $3000 \mathrm{~K}$ and disrupted over $4000 \mathrm{~K}$.
\end{abstract}




\section{Introduction}

Discovery of carbon nanotubes (CNT) [1] has stimulated both fundamental and technological interests in nanometer-scale tubular forms consisting of three-fold coordinated carbon atoms. One of the most fascinating characteristics is that the nanotubes exhibit interesting variations of their electronic structures depending on their atomic arrangement along the circumference. The electronic structures of the nanotubes are characterized by the chiral index $(n, m)$ : The nanotubes are metallic when $|n-m|$ is a multiple of three, whereas they are semiconducting otherwise $[2,3]$. The peculiar electronic property is due to an anisotropic energy band of the graphene sheet and an additional boundary condition imposed on electron states of a graphite sheet which is rolled into each nanotube. Following the early studies on CNTs, theoretical calculations pointed out that the nanometer-scale graphite flakes (graphite ribbon) which are expected to exist in growth processes of fullerenes and nanotubes exhibit interesting class of edge localized states (edge state) $[4,5,6,7]$ : At the Fermi level, some states exhibit flat dispersion in a direction along the edge in a part of the Brillouin zone (BZ). The wavefunctions of these states are mainly localized at edge carbon sites, while they are extended along an edge of the graphite ribbon. It has been demonstrated that delicate balance of electron transfers among $\pi\left(2 p_{z}\right)$ orbitals situated near edge atoms results in the flat dispersion band and the edge localized character. Existence of flat bands causes a large Fermi-level density of states that is one of major factors, possibly leading to induce magnetic ordering in itinerant electron systems. Thus, the present edge states are also expected to induce certain magnetic ordering around the edge atomic sites. First-principle electronic structure calculations on graphite ribbon with zigzag edges have indeed shown that the electron spin is polarized in one direction at one of two edges and in an opposite direction at the other edge [8, 9].

The purpose of this work is to get a theoretical insight into electronic properties and energetic stability of nanotubes with a topological line defect by using total-energy electronic-structure calculations in the density functional theory (DFT) and tightbinding molecular-dynamics (TBMD) simulations. In particular, we explore a possibility of magnetic ordering on carbon nanotubes without termination of bonding network either by vacancies or by edges. Our DFT calculations clearly show that peculiar states emerge on nanotubes with a topological line defect which consists of octagons and fused pentagons. Further, the tubes exhibit magnetic ordering that polarized electron spins are localized around the topological defect and ferromagnetically aligned along the tube axis. Calculated magnetic moment is about $0.04 \mu_{B} / \AA$ for the armchair nanotubes while $0.13 \mu_{B} / \AA$ for a zigzag nanotube. We analytically show that the flat band states posses the character of those on the graphite ribbon with zigzag edges and that the nanotubes with the topological line defects are embedded with the zigzag graphite ribbon. The nanotubes with the topological line defect are found to be thermally stable and are expected to be synthesized by implantations of $\mathrm{C}_{2}$ molecules. Although the magnetism of the carbon nanotube with the negative curvature has been reported [10], our finding 
(a)

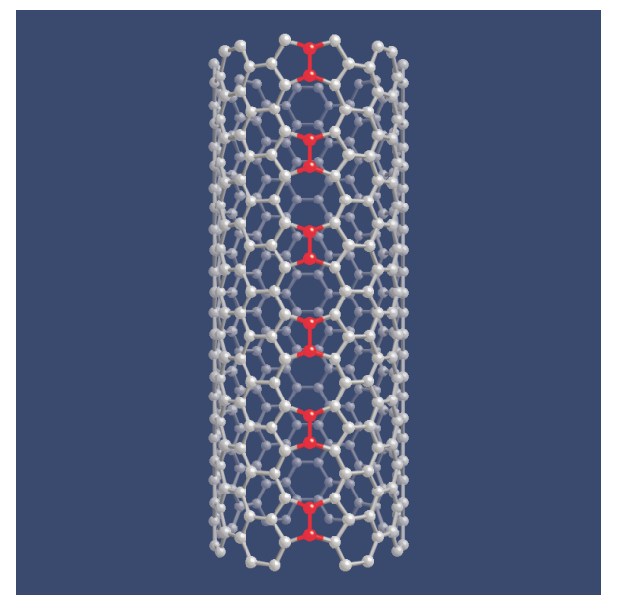

(b)

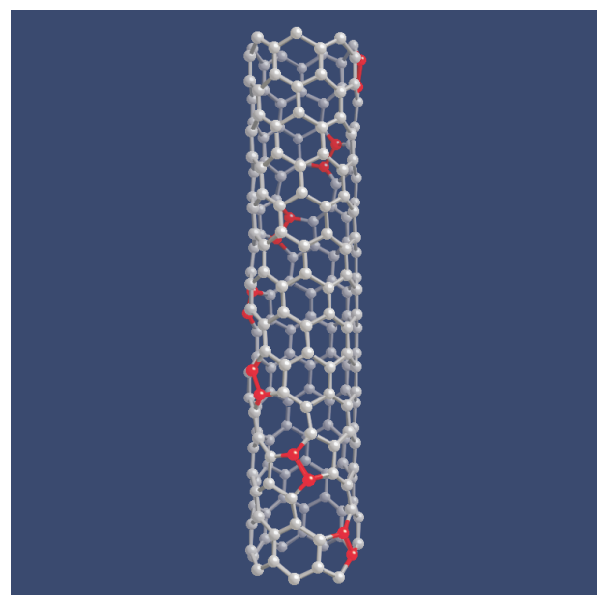

Figure 1. Optimized geometries of the (a) $(8,8)$ nanotube with the topological line defect $\left[(8,8)_{D}\right.$ nanotube] and (b) $(7,0)$ nanotube with the topological line defect $\left[(7,0)_{D}\right.$ nanotube] . Red spheres denote the $\mathrm{C}_{2}$ clusters implanted in the perfect nanotubes.

is the first evidence of a possibility of long-range spin ordering on the nanotubes with cylindrical shape consisting solely of three-fold coordinated carbon atoms.

\section{Methods}

We perform first-principles electronic-structure calculations in the framework of the density functional theory (DFT) [11, 12] using the local spin density approximation (LSDA) to elucidate the electronic and geometric structures of the topological line defect. To express the exchange-correlation energy among interacting electrons, we use a functional form [13] fitted to the Monte-Carlo results [14] for the homogeneous electron gas. Norm-conserving pseudopotentials generated by using the Troullier-Martins scheme are adopted to describe the electron-ion interaction $[15,16]$. In constructing the pseudopotentials, core radii adopted for $\mathrm{C} 2 s$ and $2 p$ states are both 1.5 Bohrs. The valence wave functions are expanded by the plane-wave basis set with a cutoff energy of $50 \mathrm{Ry}$ which is known to give enough convergence of total energy to discuss the relative stability of various carbon phases $[15,17]$. We adopt a supercell model in which a nanotube is placed with its wall being separated by $8.0 \AA$ from another wall of an adjacent nanotube. The conjugate-gradient minimization scheme is utilized both for the electronic-structure calculation and for the geometry optimization [18]. Integration over one-dimensional Brillouin zone is carried out using four $k$ points. We consider the armchair $(n, n)$ nanotubes and zigzag $(7,0)$ nanotube with the topological line defect, denoted by $(n, n)_{D}$ and $(7,0)_{D}$, respectively, in which octagons and fused pentagons are alternately aligned in parallel and spiral for $(n, n)_{D}\left[\right.$ Fig. 1 (a)] and $(7,0)_{D}$ [Fig. 1 (b)], respectively, to the tube axis. In the $(n, n)_{D}$ nanotubes, the peculiar topological defect can be obtained by the implantation of $\mathrm{C}_{2}$ cluster in a hexagon per double periodicity 
(a)

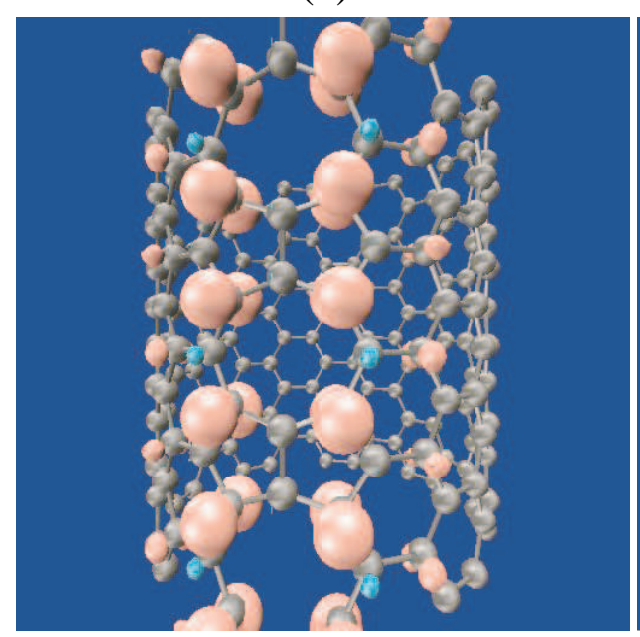

(b)

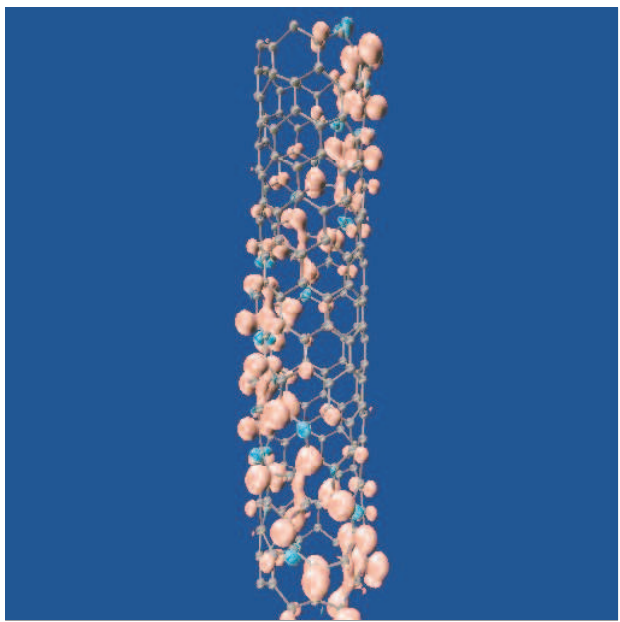

Figure 2. Isosurfaces of the spin density distribution $\left(\Delta \rho=\rho_{\uparrow}-\rho_{\downarrow}\right)$ for the nanotubes with the topological defect: (a) $(8,8)_{D}$ and $(\mathrm{g})(7,0)_{D}$ nanotubes. Pink and cyan surfaces denote the surfaces corresponding to the positive and negative density of the electron spin.

of the pristine armchair nanotubes (lattice parameter along the tube axis $c$ is $4.912 \AA$ ) [Fig. 1(a)]. On the other hand, in the $(7,0)_{D}$ nanotube, the topological line defect has a spiral arrangement in which the $\mathrm{C}_{2}$ clusters are implanted in the angle of 30 degree to the tube axis direction [Fig. 1(b)]. Owing to this spiral implantation, we take seven times periodicity of the pristine the $(7,0)$ nanotube with seven $\mathrm{C}_{2}$ clusters $(c=30.32 \AA)$. During geometry optimizations, we fixed the lattice parameter along the tube axis.

\section{Results and Discussions}

Figure 2 (a) shows the spin density $\left(\Delta \rho=\rho_{\uparrow}-\rho_{\downarrow}\right)$ of fully optimized geometry of the $(8,8)_{D}$ nanotube. Obviously, the $(8,8)$ nanotube with the topological defect exhibit magnetic ordering: Along the circumference of the nanotube, the polarized electron spins are strongly localized to the atoms connected to the $\mathrm{C}_{2}$ clusters [Fig. 1 (a)], and the distribution of the spins rapidly decreases with increasing distance from the defect. In sharp contrast, along the tube axis direction, the polarized electron spins are aligned parallel with the direction clearly exhibiting their ferromagnetic spin ordering. Thus, the armchair nanotubes with the topological line defect are ferromagnetic nanowires consisting solely of threefold-coordinated $\mathrm{C}$ atoms without carrier doping or network termination imposed by vacancies or edges.

In addition to the armchair nanotubes with the topological line defects, for the $(7,0)_{D}$ nanotube, spin density also exhibits similar characteristics to that of the $(8,8)_{D}$ nanotube: They are localized to the atoms adjacent to the implanted $\mathrm{C}_{2}$ and ferromagnetically aligned along the direction to the topological line defect resulting in spiral spin distribution [Fig. 2(b)]. Thus the ferromagnetic spin on the nanotubes 
Table 1. The magnetic moments $\mu\left[\mu_{\mathrm{B}} / \AA\right]$ of the nanotubes with topological line defects. Energy gains from non-magnetic states to the ferromagnetic ones of $(n, n)_{D}$ nanotubes $\Delta E[\mathrm{meV}]$.

\begin{tabular}{llllllll}
\hline & $(7,0)_{D}$ & $(5,5)_{D}$ & $(6,6)_{D}$ & $(7,7)_{D}$ & $(8,8)_{D}$ & $(9,9)_{D}$ & $(10,10)_{D}$ \\
\hline$\mu$ & 0.138 & 0.035 & 0.053 & 0.041 & 0.037 & 0.037 & 0.045 \\
$\Delta E$ & - & 2.9 & 6.3 & 4.2 & 3.9 & 3.8 & 2.3 \\
\hline
\end{tabular}

considered is the inherent in the nanotube possessing the topological line defects consisting of fused pentagons and octagons.

Corresponding magnetic moments are listed in Table 1. It is clear that finite magnetic moments do exist on the nanotubes with the topological line defects and that the number of polarized electron spins is insensitive to the tube diameter. Due to the spiral distribution of the polarized electron spin on the $(7,0)_{D}$ nanotube, the number of polarized electron spin is slightly larger than those on the $(n, n)_{D}$ nanotubes. The ferromagnetic states of the $(n, n)_{D}$ nanotubes are slightly lower in total energy than the non-magnetic ones. The energy gains are also shown in Table 1 . They clearly indicate that the ferromagnetic spin ordering takes place on the $(n, n)_{D}$ nanotubes below the Curie temperature of about a few tens K.

We next focus on the electronic structures of the armchair nanotubes with the topological line defect. Figure 3 (a) shows the dispersion relation of the $(8,8)_{D}$ nanotube. The electronic structure of the $(8,8)_{D}$ nanotube exhibits different characteristics from that of the pristine armchair nanotubes. Although the $(8,8)_{D}$ nanotubes have no termination of bonding network, either by vacancies or by edges, we find a flat dispersion band around the $\Gamma$ point near the Fermi level. Moreover, the flat band is split by $0.1 \mathrm{eV}$ into the majority and minority spin bands resulting in the ferromagnetic spin ordering along the tube axis. The flat bands are slightly occupied by electrons so that the Coulomb interaction among the electrons results in the band splitting and the magnetic ordering. Flat dispersion bands are usually concerned with the electron states of localized nature. However, this is not the case. As shown in Fig. 3(a), the flat dispersion band clearly looses its flatness at $k=2 \pi / 3$ and exhibits a substantial dispersion around the zone boundary. The flat band, thus, results from a delicate balance of electron transfers among the $\pi$ orbitals situated near the topological line defect. In the case of the $(7,0)_{D}$ nanotube, we find four flat dispersion bands in whole BZ, which exhibit significant exchange splitting resulting in the ferromagnetic spin ordering. It should be noted that the difference of the number of the flat band between the $(8,8)_{D}$ and the $(7,0)_{D}$ nanotubes is originated from the difference between the BZ size of them. Due to the large lattice parameter or small BZ size of the $(7,0)_{D}$ nanotube, the flat band of the edge states of the graphite ribbon are multiplicatively folded into short one-dimensional BZ of the $(7,0)_{D}$ nanotube resulting in the four flat band near the EF.

The analysis of the wave function provides an insight into the flat band states. For 
$(8,8)_{D}$ nanotube, at the $\Gamma$ point, the wave function of the flat band states is completely localized to the atoms connected to the $\mathrm{C}_{2}$ clusters [Fig. 3 (a)], while it is extended along the tube axis. The wave-function distribution is evidently different from that of the states near the flat band states: The electron states other than the flat band states are extended over the whole region of the nanotube. While the flat band loses their localized character by increasing the wave number and completely extended whole atomic site at $k=2 \pi / 3$. Similar localized character is also observed on the $(7,0)_{D}$ nanotube: The wave function of the lowest branch of flat dispersion band clearly exhibits its localized character at $\Gamma$ point [Fig. $3(b)$ ]. While the wave function of remaining three states with higher energy values lose their localized character. These three states correspond to the states at the $k$-points with finite values in single periodicity of pristine $(7,0)$ nanotube, which are folded into the $\Gamma$ point of sevenfold periodicity of $(7,0)_{D}$ nanotubes. The calculated wave function, spin densities, and energy bands thus demonstrate that the flat-band ferromagnetism is realized in the $(n, m)_{D}$ nanotubes without magnetic elements and electron/hole doping.

It is found that the nanotubes with the topological defect are energetically stable: The total energy of the nanotubes with the defect monotonically decreases with increasing the tube radius and is slightly higher by $40 \mathrm{meV} /$ atom than the perfect nanotubes with the same radius. To explore the robustness of the nanotubes under the elevated temperature, we perform the tight-binding molecular-dynamics (TBMD) simulations [19] on the $(3,3)_{D}$ nanotubes including 260 atoms. The nanotube with the topological defect is stable at the $3000 \mathrm{~K}$ for over 48 psec simulation time. Further elevating the temperature, bond breaking takes place at $4000 \mathrm{~K}$, and then the nanotubes finally collapse and fall into pieces over the temperature of $5000 \mathrm{~K}$. These thermal deformation procedure of nanotubes with the topological line defect are similar to that of defect-free nanotubes. Thus, the results indicate that the nanotubes with the topological line defect are found to be thermally stable and are expected to keep their tubular structures consisting of three-fold coordinated $\mathrm{C}$ atoms up to elevated temperature. In addition, there is a possibility of the transformation of the pentagon-octagon defect into the pentagon-heptagon defect under the bond rotation reaction. However, this rotation is hardly to take place. Our DFT calculations with the constraint minimization scheme indicate that the activation barriers for such bond rotation are about $7 \mathrm{eV}$.

\section{Summary}

In the present work, our calculations based on the density functional theory have clarified that the armchair nanotubes with the topological line defects consisting of the pentagon and octagon rings exhibit ferromagnetic spin ordering without network edges, dangling bonds, or carrier doping. The polarized electron spins are localized at the edges of the topological line defect but ferromagnetically extended along the tube direction. Calculated magnetic moment is about $0.04 \mu_{B} / \AA$. We give an analytic solution for a flat band state near the Fermi level, which induces the magnetic ordering. Based on 
(a)

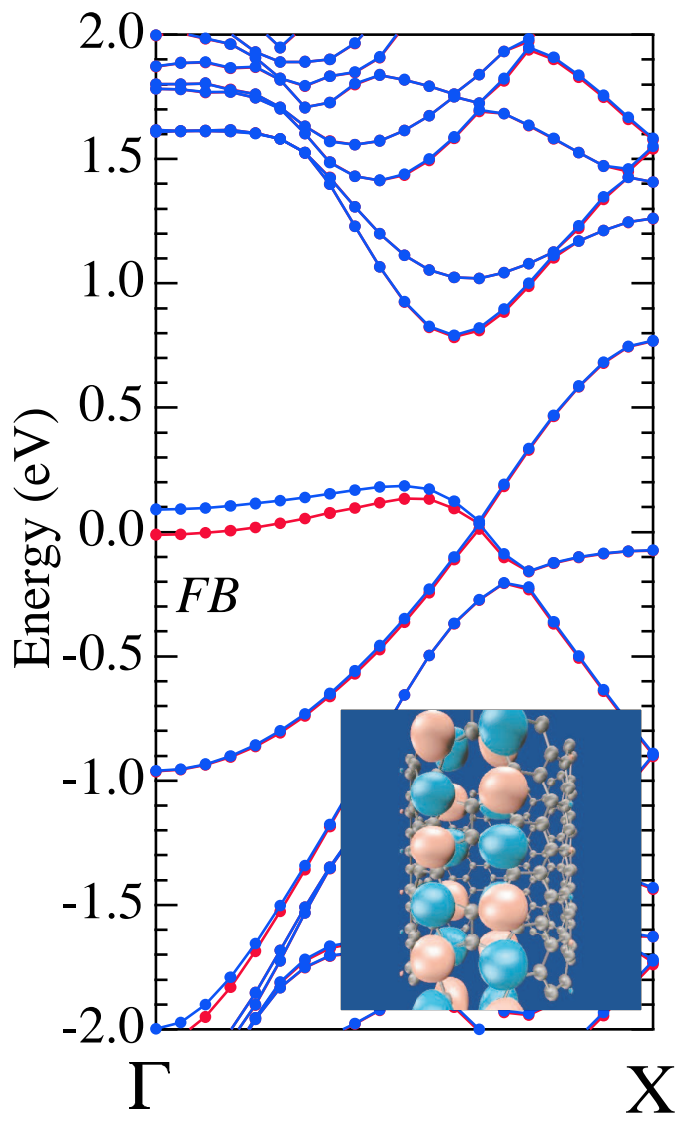

(b)

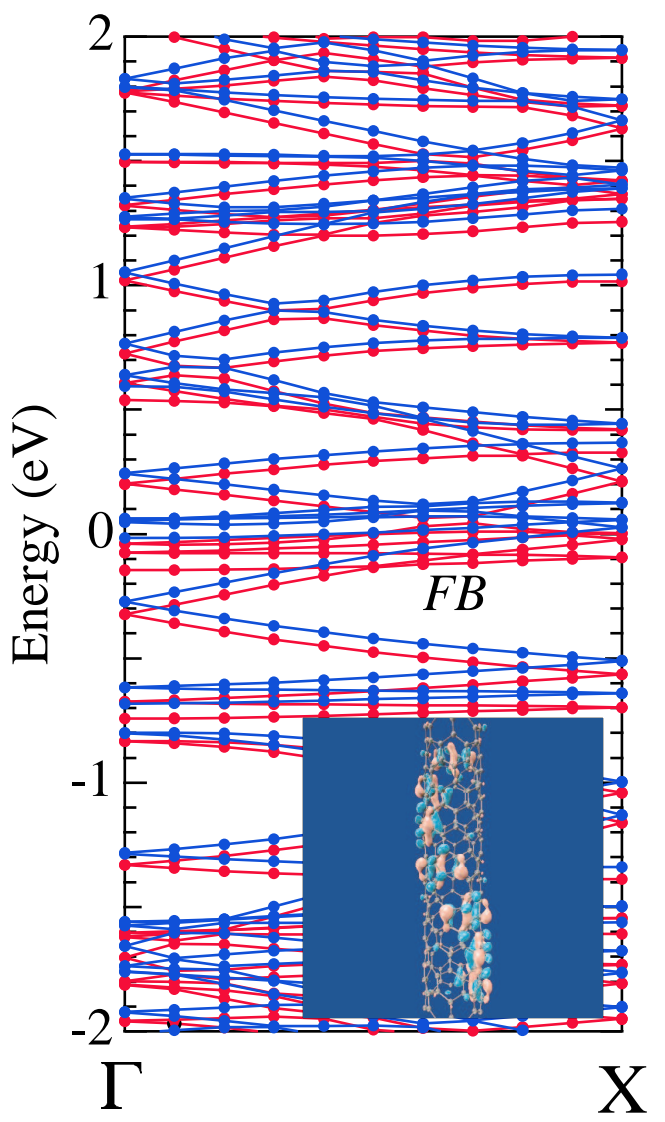

Figure 3. Electronic energy band of the $(8,8)_{D}$ and $(7,0)_{D}$ nanotubes. Blue and red lines denote the energy band for the minority and majority spins, respectively. Energies are measured from the Fermi level energy. The FB denotes the flat band state possessing edge states character. Inset: Isosurfaces of the wave functions at the $\Gamma$ point of the lowest branch of the flat band states denoted by $F B$.

the TBMD simulations, we show that the nanotubes with the topological line defect are thermally stable. The nanotubes should be synthesized by implantations of $\mathrm{C}_{2}$ molecules with appropriate indident velocity under the elevated temperature. The results give a possibility of long-range spin ordering on the $\pi$ electron networks consisting solely of three-fold coordinated carbon atoms.

\section{Acknowledgments}

We would like to thank A. Oshiyama for providing the DFT program used in this work. This work was partly supported by CREST in Japan Science and Technology Agency, a special research project on nanoscience in University of Tsukuba, and a grant-in-aid for scientific research from the Ministry of Education, Culture, Sports, Science and Technology of Japan. Computations were done at the Science Information 


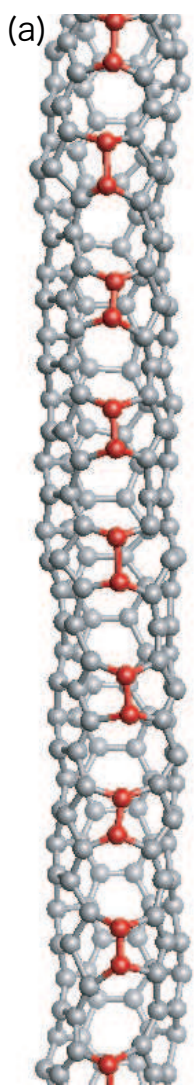

(b)

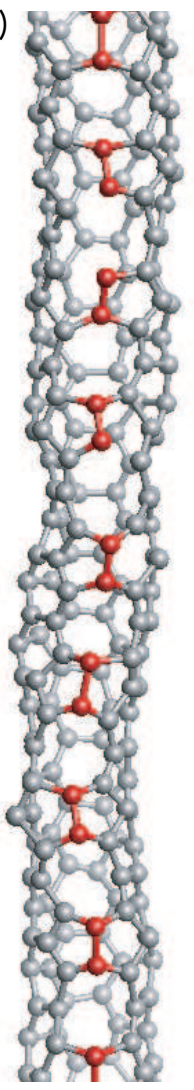

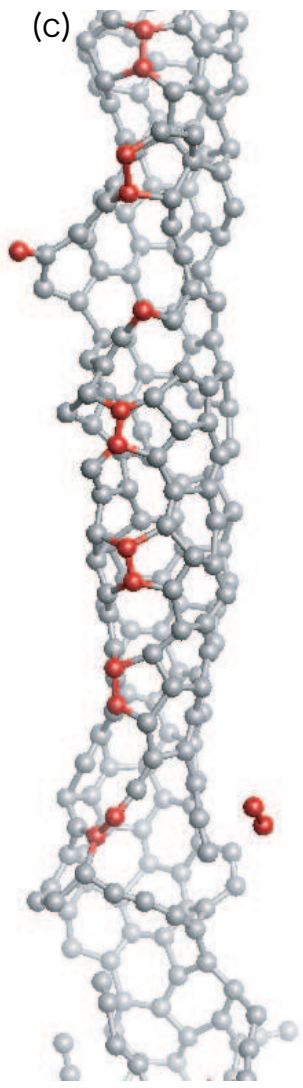

Figure 4. Geometric structures of $(3,3)_{D}$ nanotubes under the temperatures of (a) $3000 \mathrm{~K}$, (b) $4000 \mathrm{~K}$, and (c) $5000 \mathrm{~K}$. Red spheres denote the $\mathrm{C}_{2}$ clusters implanted in the perfect nanotubes.

Processing Center, University of Tsukuba, Yukawa Institute of Theoretical Physics, Kyoto University, and Research Center of the Computational Science, Okazaki National Institute.

\section{References}

[1] S. Iijima, Nature (London), 354, 56 (1991).

[2] N. Hamada, S. Sawada, and A. Oshiyama, Phys. Rev. Lett. 68 (1992) 1579.

[3] R. Saito, M. Fujita, M. S. Dresselhaus, and G. Dresselhaus, Appl. Phys. Lett. 60, 2204 (1992).

[4] M. Fujita, K. Wakabayashi, K. Nakada, and K. Kusakabe, J. Phys. Soc. Jpn. 65,1920 (1996).

[5] K. Nakada, M. Fujita, G. Dresselhaus, and M.S. Dresselhaus, Phys. Rev. B 54, 17954 (1996).

[6] Y. Miyamoto, K. Nakada, and M. Fujita, Phys. Rev. B 59, 9858 (1999).

[7] Y. Shibayama, H. Sato, T. Enoki, M. Endo, Phys. Rev. Lett. 84, 1744 (2000).

[8] S. Okada and A. Oshiyama, Phys. Rev. Lett. 87, 146803 (2003).

[9] S. Okada and A. Oshiyama, J. Phys. Soc. Japan, 72, 1510 (2003).

[10] N.-J. Park, M. Yoon, S. Berber, J. Ihm, E. Osawa, D. Tomanek, Phys. Rev. Lett. 91, 237204 (2003).

[11] P. Hohenberg and W. Kohn, Phys. Rev. 136, B864 (1964).

[12] W. Kohn and L. J. Sham, Phys. Rev. 140, A1133 (1965).

[13] J. P. Perdew and A. Zunger, Phys. Rev. B 23, 5048 (1981). 
[14] D. M. Ceperley and B. J. Alder, Phys. Rev. Lett. 45, 566 (1980).

[15] N. Troullier and J. L. Martins, Phys. Rev. B 43, 1993 (1991).

[16] L. Kleinman and D. M. Bylander, Phys. Rev. Lett. 48, 1425 (1982).

[17] S. Okada, S. Saito, and A. Oshiyama, Phys. Rev. Lett., 86, 3835 (2001).

[18] O. Sugino and A. Oshiyama, Phys. Rev. Lett. 68, 1858 (1992).

[19] C. H. Xu, C. Z. Wang, C. T. Chan, and K. M. Ho, J. Phys. Condens. Matter 4, 6047 (1992). 